\title{
sciendo
}

\section{Force and Electromyographic Responses of the Biceps Brachii after Eccentric Exercise in Athletes and non-Athletes}

\author{
by \\ Adam Kawczyński ${ }^{1}$
}

The aim of this study was to compare skeletal muscle response to elbow flexors eccentric exercise in athletes and non-athletes. A set of eccentric (ECC) exercises was performed in a group of 12 athletes and 12 non-athlete controls. Maximal isometric force, electromyographic (EMG) activity of the biceps brachii and the resting elbow angle were assessed before, immediately, 48 hours, 5 and 10 days after high-intensity ECC exercises. During the set of the ECC exercises each participant performed 25 eccentric contractions of elbow flexors. Each contraction consisted of lowering a dumbbell from the flexed (elbow joint angle: 50) to the extended elbow (elbow joint angle: 180) position. The weight of the dumbbell was set at $80 \%$ of one-repetition maximum (1RM). The ECC contractions caused a decrease in maximal isometric force in both groups. The variable dropped by $8 \%$ in non-athletes and by $24 \%$ in athletes. Furthermore, the EMG RMS increased significantly only for non-athletes 10 days after the ECC exercise compared to baseline values. The present study showed different effects of ECC exercise on force and EMG in athletes and nonathletes, indicating a more pronounced force response in athletes and electromyographic response in non-athletes.

Key words: athletes, force, electromyography, eccentric contraction.

\section{Introduction}

Eccentric (ECC) exercise is a specific type of muscle work. Fast twitch motor units are the main units recruited during ECC exercise (Nardone and Schieppati, 1988), therefore, maximal force generated during eccentric contractions is about $30-40 \%$ higher than during concentric contractions (Komi and Rusko, 1974). The main effects of ECC exercise are prolonged strength loss, muscle fibers damage, delayed onset muscle soreness, and muscle shortening resulting in a decreased joint angle (Clarkson et al., 1992).

It is well known that unaccustomed ECC exercise induces several mechanical and functional changes in muscles. Most of the research on ECC exercise has been carried out on non-athletes or those who have not been involved in resistance training programs (Kawczynski et al., 2018). Recent studies, which focused on non- athletes, showed that training can modify response to ECC exercise. An experiment performed by Rinard and co-workers (2000) proved that previous concentric training caused greater changes in measured variables and suggested increased vulnerability to eccentric exercise-induced responses and muscle injury (Rinard et al., 2000). On the contrary, Nosaka and Newton (2002) showed that previous concentric training did not exacerbate eccentric exerciseinduced muscle damage.

The influence of a single bout of ECC exercise performed by previously untrained subjects is well documented. Either maximal or submaximal bouts of ECC exercise attenuate muscle damage after subsequent ECC exercise. This protective effect lasts from 1 to 10 weeks after initial ECC exercise, and in recent literature is called a "repeated bout effect" (Chen et al., 2019).

\footnotetext{
1 - University School of Physical Education, Faculty of Sport Sciences, Department of Paralympics Games, 51-629 Wroclaw, ul. Paderewskiego 35, Poland.
} 
Newton et al. (2008) showed that subjects involved in strength training (at least 1 year of training) presented different responses to ECC exercise compared to untrained subjects. In that experiment changes in maximal voluntary isometric and isokinetic torque, range of motion, plasma creatine kinase activity, and muscle soreness before, immediately and 5 days after ECC exercise were compared between groups. The trained group showed significantly smaller changes in all of the measures except for muscle soreness and faster recovery of muscle function compared with untrained subjects (Newton et al., 2008).

Athletic training causes muscle adaptation. Adaptive changes include increases in maximal isometric force (MIF), a higher rate of force development, shortening of the contraction time, muscle hypertrophy, changes in motor unit recruitment pattern and changes in muscle mechanomyographic and electromyographic activity (Evetovich et al., 2000; Maszczyk et al., 2016; Golas et al., 2018). Increases in maximal force, muscle electromyography (EMG) amplitude and the rate of EMG activation of knee extensors, as the effect of sport-specific resistance training in well-trained track and field athletes, have been previously reported. Judge et al. (2003) suggest that these changes, especially in EMG variables indicate that improvements in the pattern of neural drive occur along with sport-specific resistance training. In the group that consisted of 15 untrained subjects, 14 weeks of heavyresistance training resulted in increases in maximal force, the rate of force development and EMG amplitude. These findings could be explained by enhanced neural drive (Aagaard et al., 2002).

These examples show that ECC exercise research has several aspects: a) acute and long term effects of ECC exercise; b) effects of various types of muscle contractions (concentric vs. eccentric) on muscle response after subsequent ECC exercise; c) comparison of responses to ECC exercise in trained and untrained individuals.

Considering the presented studies, it seems reasonable to investigate competitive athletes to extend knowledge in this area. Therefore, the aim of this study was to compare force and EMG responses to elbow flexors ECC exercise in athletes and non-athletes.

\section{Methods}

\section{Participants}

Twenty-four individuals participated in the present study. The experimental group consisted of 12 athletes, karate competitors (aged $28.6 \pm 7.8$ years; body height $182.3 \pm 5.2 \mathrm{~cm}$; body mass $85.0 \pm 7.4 \mathrm{~kg}$ ) and 12 non-athletes, students (aged $22.2 \pm 2.5$ years; body height $180.3 \pm 6.3 \mathrm{~cm}$; body mass $74.1 \pm 6.6 \mathrm{~kg}$ ). Informed consent was obtained from each participant. Athletes were in the off-season and all study participants maintained their normal daily activities. The study was approved by the ethics committee of the University School of Physical Education in Wrocław.

\section{Experimental Sessions}

The experiment consisted of 5 sessions. During the first session, at which written informed consent was provided, participants were familiarized with experimental procedures. Additionally, maximal isometric force (MIF) on a BIODYNA dynamometer was assessed (Jaskolska et al., 2004). The participants also performed a one-repetition maximum test (1RM) of the concentric curl dumbbell exercise. The rest interval between MIF and 1RM assessment was 15 minutes. The first session was followed by 10 days of recovery. During the second session the ECC exercise protocol was performed. The third session took place 48 hours after the ECC exercise protocol while the fourth and fifth sessions began 5 and 10 days after the ECC exercise protocol, respectively.

\section{Eccentric exercise protocol}

ECC exercise consisted of lowering a dumbbell from the flexed (elbow join angle: $50^{\circ}$ ) to the extended elbow (elbow join angle: $180^{\circ}$ ) position. The dumbbell weight was set at $80 \%$ of 1RM.

Each participant performed 25 eccentric repetitions. Each lowering of the dumbbell lasted $5 \mathrm{~s}$ and the investigator brought the dumbbell up to the flexed position after each eccentric repetition. The investigator's help was needed to avoid concentric contractions of the elbow flexors. During ECC exercise participants were seated on a chair with their back supported. The participant's trunk was fixed to the chair by chest belts. The right arm was supported by a specially designed cushioned support to stabilize the elbow during the ECC exercise. The left arm was placed 
on the left thigh (Jaskolska et al., 2004).

\section{Repetition maximum procedures}

The assessment of the one-repetition maximum (1RM) dumbbell load was carried out on the same support as the performance of ECC exercises. The participant's position during testing was the same as during the ECC exercise. The maximal dumbbell load that each participant could lift from elbow full extension to elbow full flexion, without any assistance, was taken as the 1RM. The warm-up consisted of two sets of 8 repetitions with a $4 \mathrm{~kg}$ dumbbell. Following the warm up participants performed three maximal attempts. The warm-up and maximal attempts were separated by 3 min rest intervals.

\section{Maximal isometric force procedures}

A BIODYNA dynamometer was used in the present experiment (designed by Warsaw Technical University, Poland) (Jaskolska et al., 2004). The participants were seated on a chair with their back supported, and with the right arm abducted to $90^{\circ}$ and flexed to $90^{\circ}$ in the elbow joint. The forearm was positioned on the BIODYNA dynamometer in the neutral position between pronation and supination. The participant's trunk was fixed to the chair by chest belts.

During MIF procedures participants were instructed to react to signals generated by the computer. When participants heard the first signal, they had to flex the forearm as hard and fast as possible. Two to three seconds later, the next signal was emitted and the participant had to relax immediately.

Emg recording

The EMG electrode used in this study was built into the custom made EMG/MMG probe, which has been described in details in previous research (Jaskolska et al., 2004).

EMG electrodes were used to detect electrical activity of the biceps brachii. Myoelectric signals were detected with bipolar, single differentiated active EMG electrodes $(\mathrm{Ag} / \mathrm{AgCl})$, of $4 \mathrm{~mm}$ in diameter and $25 \mathrm{~mm}$ apart. EMG electrodes were positioned on the midline of the biceps brachii short head, where the biggest EMG amplitude was recorded, with the detection EMG surface oriented perpendicularly to the length of the muscle belly. The places where electrodes were attached were marked on the skin with a water-proof marker so that the electrodes could be accurately repositioned each day with a placement error not greater than $\pm 1 \mathrm{~mm}$ in either direction (Jaskolska et al., 2004).

The EMG signals were amplified 500 times $(54 \mathrm{~dB})$ with a low-noise (high-input impedance) differential preamplifier built within a custom-made EMG/MMG probe (noise $18 \mathrm{nV} / \sqrt{\mathrm{Hz}}$ at $\mathrm{f}=1 \mathrm{kHz} ; \mathrm{CMRR}=$ common-mode rejection ratio $>100 \mathrm{~dB}$; THD $=$ total harmonic distortion $0.003 \%$ ). Then, the signal was amplified with a programmable gain amplifier. The gain of the EMG signal was 500-10,000 with 10-10,000 Hz. The signals were sampled at the frequency of $10,000 \mathrm{~Hz}$ per channel, and digitized by a $14 \mathrm{bit}$ A/D converter (Analog Devices) with the conversion time of $5.2 \mu$ s. The EMG signals recorded were filtered with a software low-pass filter of $500 \mathrm{~Hz}$. The RMS was used to measure the amplitude of EMG signals (Jaskolska et al., 2004).

Resting elbow angle and muscle pain/soreness asessing

The resting elbow angle was measured in a standing position using a digital goniometer. The muscle pain (soreness) intensity was assessed by means of a $10 \mathrm{~cm}$ visual analogue scale, where 0 indicated "no pain" and 10 indicated "maximal pain intensity".

Both measures were performed before, immediately, 48 hours, 5 and 10 days after the high-intensity ECC exercises.

\section{Statistical analyses}

EMG data were analyzed using repeatedmeasures analysis of variance (RMANOVA) between sessions (before, immediately after, 48 hours after, 5 and 10 days after) and between groups (athletes and non-athletes). RMANOVA was used to analyze maximal isometric force, the elbow angle and muscle pain/soreness between sessions and between groups. The Bonferroni adjustment for post hoc multiple comparisons was used. The normality of the data distribution was checked by the Shapiro-Wilk test. In all tests, $p \leq 0.05$ was considered significant. The data are presented as means and SD in the text and figures.

\section{Results}

In athletes and non-athletes MIF decreased significantly $(p \leq 0.05)$ immediately after ECC exercise as compared to baseline conditions. MIF dropped by $24 \%$ in athletes and 
by $8 \%$ in non-athletes. The drop difference between the groups was statistically significant ( $p$ $\leq$ 0.05) (Figure 1). The EMG RMS increased significantly only for non-athletes 10 days after ECC exercise compared with baseline values ( $p \leq$ 0.05) (Figure 2).

Muscle pain/soreness measured in the relaxed muscle increased significantly from before to immediately after exercise and from before to 48 hours after ECC exercise in both groups ( $p \leq$ 0.05). Muscle soreness measured during isometric contraction rose considerably from before to immediately after exercise and from before to 48 hours after ECC exercise in athletes $(p \leq 0.05)$. It was 5 days and 10 days after ECC exercise when muscle pain/soreness returned to the baseline level in athletes. In non-athletes muscle pain/soreness measured during isometric contraction increased significantly during all sessions (immediately after, 48 hours after, 5 day after and 10 days after) compared to baseline conditions $(p \leq 0.05)$.

The resting elbow angle in athletes and nonathletes decreased significantly $(p \leq 0.05)$ immediately after and 48 hours after the ECC exercise compared with initial values.

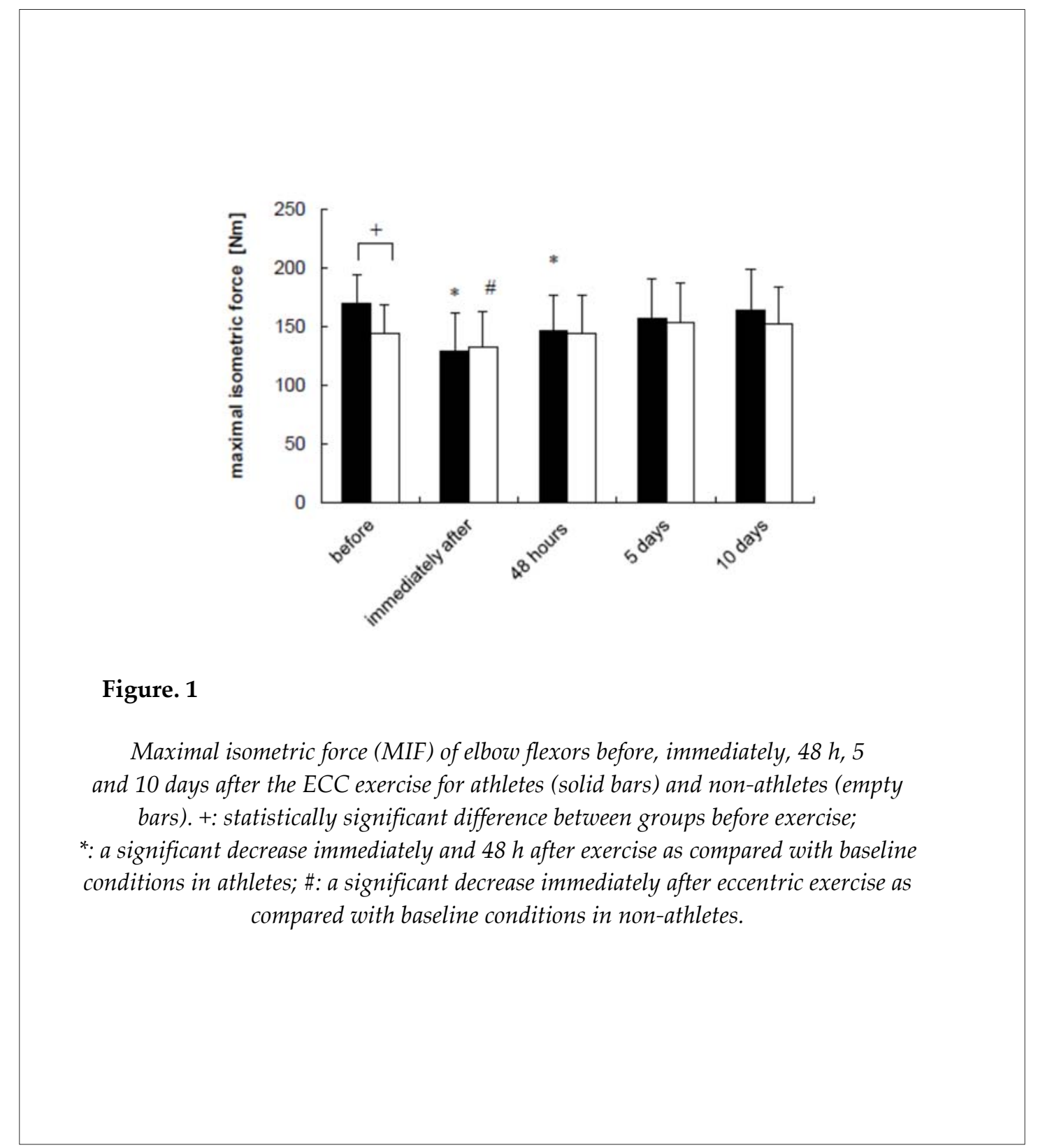




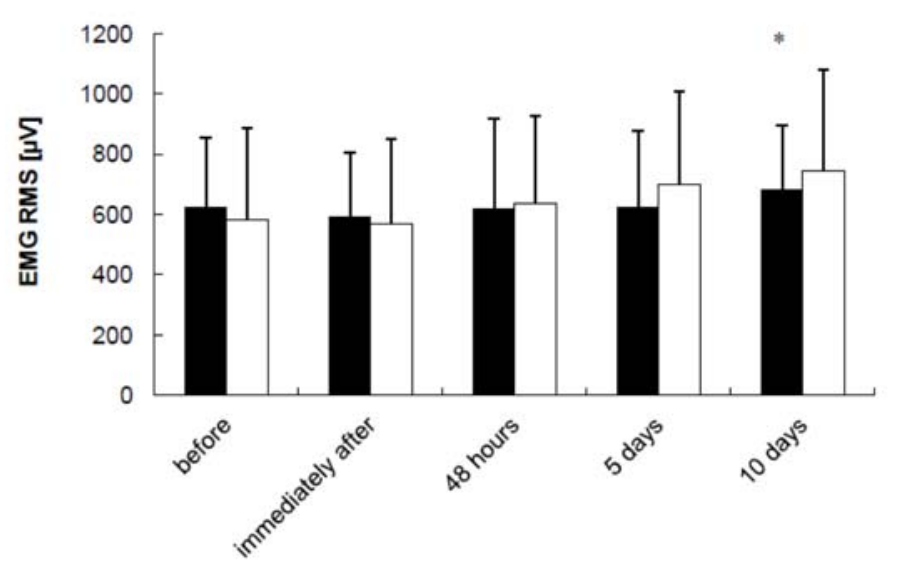

Figure. 2

Root mean square (RMS) for electromyography (EMG) before, immediately, 48 hours,

5 and 10 days after eccentric exercise for athletes (solid bars) and non-athletes

(empty bars). *: a significant increase 10 days after as compared with baseline values in non-athletes.

\section{Discussion}

\section{Maximal isometric force}

A decrease in maximal isometric force, as the effect of ECC exercise, has been documented in the literature. It has been found that the lowered force is maintained from 6-7 (Golden and Dudley 1992) to 33-47 (Sayers et al., 2003) days after ECC exercise.

Profound loss in voluntary forceproducing capability after ECC exercise is considered one of the best indirect indicators of muscle damage in humans (Warren et al., 1999). It has also been reported that this indicator is highly variable among subjects, even if they are exposed to an identical exercise protocol. Clarkson and coauthors (2005) found that among 157 subjects who completed 50 maximal lengthening actions of the elbow flexors, the response ranged from no strength loss to more than $90 \%$ strength loss immediately after the completion of the exercise protocol. Those findings indicate that the drop in MIF, also observed in our experiment, could have been caused by many factors, which has to be considered during analysis of the results.

In the present study the MIF decrease was greater in athletes than in students. These results are unexpected, and three different explanations can be provided to explain this phenomenon. First, athletes have a tendency to overload muscle fibres, which leads to a greater MIF decrease as an effect of fatigue. Second, "more selective" (not shown by EMG) fast motor unit recruitment in 
athletes causes their disruptions. Finally, athletes can withstand a dumbbell load at a full range of motion, thus muscles were loaded near their maximal length. Muscle fibers are more susceptible to damage at higher length (Newham et al., 1988).

The level of loads used during ECC exercise is also an important factor influencing the drop in MIF. This was documented by Nosaka and Newton (2002) where subjects performed ECC exercise with different loads. When the load was set at $50 \%$ of maximal isometric force, immediately after the ECC exercise the force decreased by $39 \%$. After the ECC exercise with $100 \% \mathrm{MIF}$, the force decreased by $53 \%$ compared to baseline measurements. The difference between these force decrements was statistically significant.

It should be underlined that unexpected differences in the MIF decrease between athletes and students may be an effect of the applied methodology. Factors which can affect the MIF decrease include: 1) different physical activity profiles of subjects; 2) ECC exercise performed on a cushioned support which could change the real load; 3) the cushioned support could also affect the range of motion, especially in the group of students; 4) $80 \%$ of maximal isometric force during the ECC exercise may be excessive for the weakest students and not sufficient for the strongest athletes.

\section{EMG after eccentric exercise}

In the group of athletes, EMG RMS did not change significantly after the ECC exercise, while in the group of non-athletes EMG RMS showed a tendency to increase 5 days after the exercise and reached maximum values 10 days after the ECC exercise. The results achieved in non-athletes can be an effect of changes in sarcolemma depolarization and the recruitment pattern of muscle fibers after the ECC exercise. Moreover, the present results are in line with the study of Saxton and co-workers (1995) where subjects performed 50 maximal eccentric contractions of the elbow flexors. The EMG RMS increase was accompanied by a rise in the creatine kinase activity, muscle tremor and a decrease in the elbow angle and maximal force.

\section{Muscle pain/soreness}

The presented results on muscle pain/soreness are consistent with previous experiments in that field (Chen et al., 2019; Nie et al., 2005). The present study showed, for the first time, a difference in the time pattern of muscle pain/soreness development in athletes and nonathletes. The muscle pain/soreness returned to the baseline level only in the group of athletes, which can be explained by muscle chronic adaptation to resistance training (Evetovich et al., 2000).

\section{Resting elbow angle}

Several studies have reported that after ECC exercise muscles spontaneously shorten and that the shortening is not caused by an increase in electrical activity (Howell et al., 1985). Brody (1969) presented a case study in which a patient experienced exercise-induced contractures in muscles that were electrically normal at rest. Biopsy analysis showed a decrease in $\mathrm{Ca}^{2+}$ uptake/mg of microsomal protein, and the author suggested a deficit in the ability of sarcoplasmic reticulum to sequester $\mathrm{Ca}^{2+}$ ions.

It has been also reported that muscle shortening after ECC exercise is due to an accumulation of $\mathrm{Ca}^{2+}$ in damaged fibers (Clarkson and Tremblay 1988).

Moreover, Jones and co-workers (1987) observed that muscle stiffness and spontaneous flexion at the elbow developed immediately after an ECC exercise of elbow flexors. The authors did not find significant changes in resting EMG activity and concluded that stiffness and spontaneous flexion might be a result of connective tissue damage causing subsequent sensitizing of pain receptors. Howell and coauthors (1985) indicated that flexion deformities were not accompanied by an increase in electrical activity. They concluded that oedema within the perimuscular connective tissue might alter the elastic behaviour of the muscles and cause restriction of motion. Decreases in the elbow angle due to muscle shortening can be also explained by an abnormal accumulation of $\mathrm{Ca}^{2+}$ in cells. The shortening could exert a stretch on the connective tissue and tendons, perhaps making receptors more sensitive to pain/soreness (Clarkson and Tremblay, 1988). Decreases in the elbow angle in our experiment were also caused by the above mentioned mechanisms.

In conclusion, it should be underlined that athletes and students showed a different response to ECC exercise. The main differences were: i) a greater maximal isometric force 
decrement in athletes immediately after the ECC exercise; ii) a significant increase in EMG RMS only for non-athletes 10 days after the ECC exercise as compared with baseline values; iii) a more rapid decrease of muscle pain/soreness in athletes. These findings may be used by strength and conditioning professionals as clues for injury prevention and training load programming.

\section{References}

Aagaard P, Simonsen EB, Andersen JL, Magnusson P, Dyhre-Poulsen P. Increased rate of force development and neural drive of human skeletal muscle following resistance training. J Appl Physiol, 2002; 93(4): 1318-1326

Brody IA. Muscle contracture induced by exercise. A syndrome attributable to decreased relaxing factor. $N$ Engl J Med, 1969; 281(4): 187-192

Chen TC, Yang TJ, Huang MJ, Wang HS, Tseng KW, Chen HL, Nosaka K. Damage and the repeated bout effect of arm, leg, and trunk muscles induced by eccentric resistance exercises. Scand J Med Sci Sports, 2019; 29(5): 725-735

Clarkson PM, Hoffman EP, Zambraski E, Gordish-Dressman H, Kearns A, Hubal M, Harmon B, Devaney JM. ACTN3 and MLCK genotype associations with exertional muscle damage. J Appl Physiol (1985), 2005; 99(2): 564-569

Clarkson PM, Nosaka K, Braun B. Muscle function after exercise-induced muscle damage and rapid adaptation. Med Sci Sports Exerc, 1992; 24(5): 512-520

Clarkson PM, Tremblay I. Exercise-induced muscle damage, repair, and adaptation in humans. J Appl Physiol (1985), 1988; 65(1): 1-6

Evetovich TK, Housh TJ, Weir JP, Housh DJ, Johnson GO, Ebersole KT, Smith DB. The effect of leg extension training on the mean power frequency of the mechanomyographic signal. Muscle Nerve, 2000; 23(6): 973-975

Golas A, Maszczyk A, Stastny P, Wilk M, Ficek K, Lockie RG, Zajac A. A New Approach to EMG Analysis of Closed-Circuit Movements Such as the Flat Bench Press. Sports (Basel), 2018 6(2) doi: 10.3390/sports6020027

Golden CL, Dudley GA. Strength after bouts of eccentric or concentric actions. Med Sci Sports Exerc, 1992; 24(8): 926-933

Howell JN, Chila AG, Ford G, David D, Gates T. An electromyographic study of elbow motion during postexercise muscle soreness. J Appl Physiol (1985), 1985; 58(5): 1713-1718

Jaskolska A, Brzenczek W, Kisiel-Sajewicz K, Kawczynski A, Marusiak J, Jaskolski A. The effect of skinfold on frequency of human muscle mechanomyogram. J Electromyogr Kinesiol, 2004; 14(2): 217-225

Jones DA, Newham DJ, Clarkson PM. Skeletal muscle stiffness and pain following eccentric exercise of the elbow flexors. Pain, 1987; 30(2): 233-242

Judge LW, Moreau C, Burke JR. Neural adaptations with sport-specific resistance training in highly skilled athletes. J Sports Sci, 2003; 21(5): 419-427

Kawczynski A, Mroczek D, Andersen RE, Stefaniak T, Arendt-Nielsen L, Madeleine P. Trapezius viscoelastic properties are heterogeneously affected by eccentric exercise. J Sci Med Sport, 2018; 21(8): 864-869

Komi PV, Rusko H. Quantitative evaluation of mechanical and electrical changes during fatigue loading of eccentric and concentric work. Scand J Rehabil Med Suppl, 1974; 3(121-126

Maszczyk A, Golas A, Czuba M, Krol H, Wilk M, Stastny P, Goodwin J, Kostrzewa M, Zajac A. EMG Analysis and Modelling of Flat Bench Press Using Artificial Neural Networks. SAJRSPER, 2016; 38(1): 91-103

Nardone A, Schieppati M. Shift of activity from slow to fast muscle during voluntary lengthening contractions of the triceps surae muscles in humans. J Physiol, 1988; 395: 363-381

Newham DJ, Jones DA, Ghosh G, Aurora P. Muscle fatigue and pain after eccentric contractions at long and short length. Clin Sci (Lond), 1988; 74(5): 553-557 
Newton MJ, Morgan GT, Sacco P, Chapman DW, Nosaka K. Comparison of responses to strenuous eccentric exercise of the elbow flexors between resistance-trained and untrained men. J Strength Cond Res, 2008; 22(2): 597-607

Nie H, Kawczynski A, Madeleine P, Arendt-Nielsen L. Delayed onset muscle soreness in neck/shoulder muscles. Eur J Pain, 2005; 9(6): 653-660

Nosaka K, Newton M. Difference in the magnitude of muscle damage between maximal and submaximal eccentric loading. J Strength Cond Res, 2002; 16(2): 202-208

Rinard J, Clarkson PM, Smith LL, Grossman M. Response of males and females to high-force eccentric exercise. J Sports Sci, 2000; 18(4): 229-236

Saxton JM, Clarkson PM, James R, Miles M, Westerfer M, Clark S, Donnelly AE. Neuromuscular dysfunction following eccentric exercise. Med Sci Sports Exerc, 1995; 27(8): 1185-1193

Sayers SP, Knight CA, Clarkson PM. Neuromuscular variables affecting the magnitude of force loss after eccentric exercise. J Sports Sci, 2003; 21(5): 403-410

Warren GL, Lowe DA, Armstrong RB. Measurement tools used in the study of eccentric contractioninduced injury. Sports Med, 1999; 27(1): 43-59

\section{Corresponding author:}

\section{Adam Kawczyński}

University School of Physical Education, Faculty of Sport Sciences,

Department of Paralympics Games,

51-629 Wroclaw,

ul. Paderewskiego 35, Poland

E-mail: kawczynski.a@gmail.com 